\title{
The unique field-of-view and focusing budgets of PLATO
}

\section{Martin Pertenais, Juan Cabrera, Carsten Paproth, Anko Boerner, Denis Grießbach, et al.}

Martin Pertenais, Juan Cabrera, Carsten Paproth, Anko Boerner, Denis Grießbach, Valery Mogulsky, Heike Rauer, "The unique field-of-view and focusing budgets of PLATO," Proc. SPIE 11852, International Conference on Space Optics - ICSO 2020, 118524Y (11 June 2021); doi: $10.1117 / 12.2599820$ SPIE. Event: International Conference on Space Optics - ICSO 2021, 2021, Online 


\section{International Conference on Space Optics-ICSO 2020}

Virtual Conference

30 March-2 April 2021

Edited by Bruno Cugny, Zoran Sodnik, and Nikos Karafolas
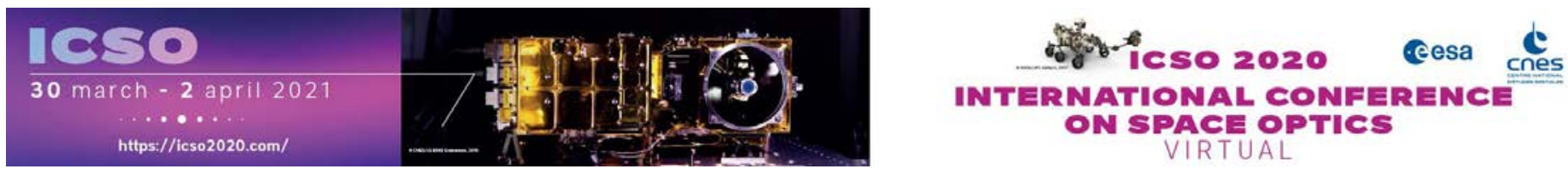

\section{The unique field-of-view and focusing budgets of PLATO}

\section{Cesa isopmeatians ecnes}




\title{
The unique field-of-view and focusing budgets of PLATO
}

\author{
Martin Pertenais ${ }^{\mathrm{a}}$, Juan Cabrera ${ }^{\mathrm{b}}$, Carsten Paproth ${ }^{\mathrm{a}}$, Anko Boerner ${ }^{\mathrm{a}}$, Denis Grießbach ${ }^{\mathrm{a}}$, \\ Valery Mogulskyc ${ }^{\mathrm{c}}$, and Heike Rauer ${ }^{\mathrm{b}, \mathrm{d}, \mathrm{e}}$ \\ ${ }^{a}$ German Aerospace Center (DLR), Institute for Optical Sensor Systems, Rutherfordstr. 2, \\ 12489 Berlin, Germany \\ ${ }^{\mathrm{b}}$ German Aerospace Center (DLR), Institute for Planetary Research, Rutherfordstr. 2, 12489 \\ Berlin, Germany \\ ${ }^{\mathrm{c}}$ OHB System AG, Manfred-Fuchs-Straße 1, 82234 Weßling, Germany \\ ${ }^{\mathrm{d}}$ Institute of Geological Sciences, Freie Universität Berlin, Malteserstr. 74-100, D-12249 Berlin, \\ Germany \\ ${ }^{\text {e}}$ Department of Astronomy and Astrophysics, Berlin University of Technology, Hardenbergstr. \\ 36, D-10623 Berlin, Germany
}

\begin{abstract}
The PLAnetary Transits and Oscillations of stars mission (PLATO) is the M3 mission in ESA's Cosmic Vision 2015-2025 Programme, see Rauer et al. (2014). ${ }^{1}$ The PLATO mission aims at detecting and characterizing extrasolar planetary systems, including terrestrial exoplanets around bright solar-type stars in the habitable zone. In order to achieve its scientific objectives, PLATO must perform uninterrupted high precision photometric monitoring of large samples of stars during long periods to detect and characterize planetary transits. The scientific payload of PLATO, developed and provided by the PLATO Mission Consortium (PMC) and ESA, is based on a multi-telescope configuration consisting of 24 "Normal" (N) cameras and 2 "Fast" (F) cameras, so as to provide simultaneously a large field of view and a large collecting aperture. The optical design is identical for all cameras and consists of a 6-lens dioptric design with a $120 \mathrm{~mm}$ entrance pupil and an effective field of view of more than $1000 \mathrm{deg} 2$. This concept results in an overall field-of-view of more than $2000 \mathrm{deg}^{2}$, spread over 104 CCDs of 20 mega-pixels each. Associated to very accurate pointing and alignment requirements, this is a real challenge to define and breakdown precise specifications to several sub-systems in order to ensure that this overall field of view budget is achieved and verified. We propose to go through the budget that was performed for the PLATO camera and to describe how we intend to satisfy this scientific requirement. To make it more challenging, it has to be taken into account that the PLATO spacecraft will have to rotate of $90^{\circ}$ every three months without changing its field of view (due to its orbit in L2 and the sun illumination limitations). This has to be considered in the breakdown of the budget and design of all sub-systems. A consequence of this large field of view is the difficulty to reach very good and harmonious optical performances across the field, and in a realistic depth of focus. Therefore, the focusing budget is also very challenging for the development of the PLATO cameras. We will describe the way the PLATO's camera focusing budget has been broken down into allocations and how it is planned to be verified. To ensure optimal performances in-flight, the PLATO cameras have the extraordinary capabilities to perform re-focusing using a high precision Thermal Control System (TCS). Each individual camera on the payload can be thermally controlled independently from its neighbor to reach its own optimal operational temperature. The different consequences of this concept into the budget allocations and sub-system development will be discussed.
\end{abstract}

Keywords: PLATO, exoplanet, field-of-view, focusing

\section{PLATO PAYLOAD CONCEPT}

\subsection{Camera Arrangement on the Spacecraft}

The 26 cameras composing PLATO payload are arranged on the spacecraft in 5 different groups:

Further author information: (Send correspondence to M. Pertenais: e-mail: martin.pertenais@dlr.de 
- the 2 Fast Cameras (F-CAMs, red in the left picture of Fig. 1) are placed at the top of the optical bench and will have a pointing direction parallel to the $\mathrm{Z}$ axis of the Payload Module (PLM) on the spacecraft, as well as to the mean boresight of all other cameras.

- the 24 Normal Cameras (N-CAMs) are split in 4 groups of 6 . Within one group, the 6 cameras are coaligned and will point to the same field of view (FoV). Each group is tilted by $9.2^{\circ}$ compared to the F-CAMs (or $Z_{P L M}$ ), as shown in the right sketch of Fig. 1.
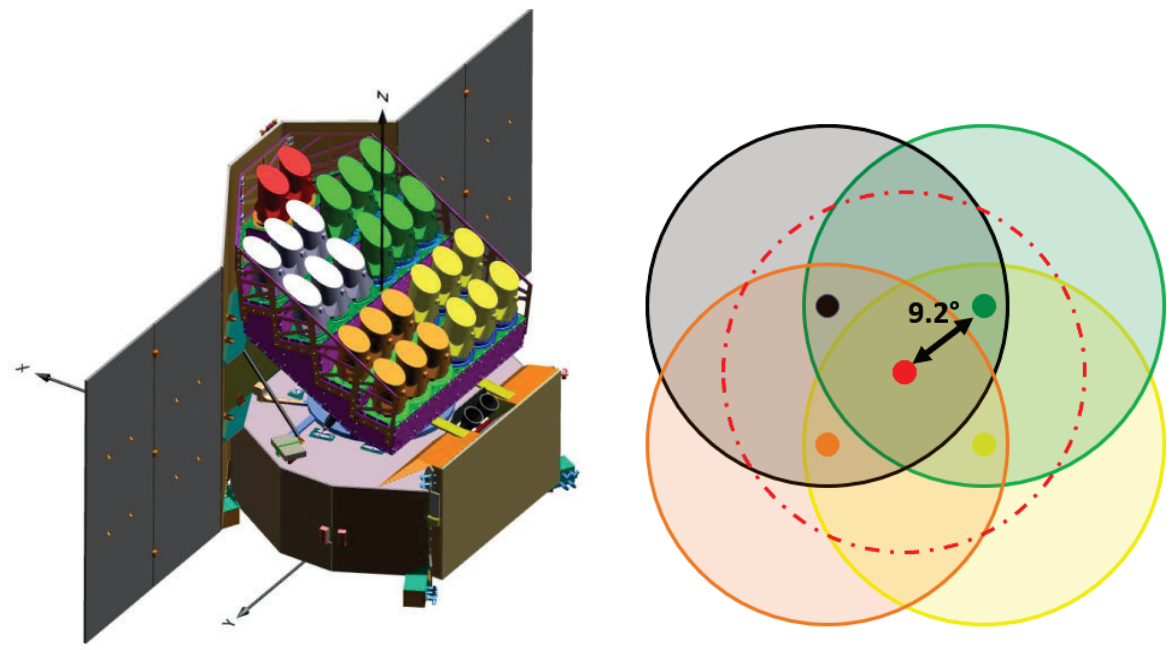

Figure 1. Left: Group arrangement of the cameras on the spacecraft. Credits: OHB System AG. Right: Tilt Angle of $9.2^{\circ}$ for each of the $4 \mathrm{~N}-\mathrm{CAM}$ groups.

The full detailed mathematical description of the coordinate matrices transformation between each CAM group and the PLM reference frame is described in Appendix. A.

The configuration of the CAM group on the spacecraft is the outcome of a trade-off, ${ }^{2}$ balancing the global field of view of PLATO and the final signal to noise ratio (SNR) of the stars observed. The SNR of a star indeed increases with the square root of the number of cameras that have this star in their FoV. Optimally, to maximize the SNR we would need a group angle of $0^{\circ}$. Having all $24 \mathrm{~N}-\mathrm{CAMs}$ pointing at the same exact FoV is indeed maximizing the SNR budget as all stars would be observed by all CAMs. On the other side, we also want at the same time to maximize the number of stars observed by having the largest FoV possible, meaning the largest angle between the group as possible. Both objectives are pulling in the opposite direction and is the source of this trade-off need. As shown in this paper, the chosen configuration with 4 groups of 6 CAMs and $9.2^{\circ}$ angle between a group and the F-CAM boresight provides optimal performance for both field of view and for SNR.

\subsection{Quarterly Rolls Operations Procedure}

PLATO satellite will operate from an elliptical orbit at the Lagrange point L2, at around 1.5 million kilometers from Earth in the opposite direction from the Sun. The basic operation strategy of PLATO is to stare at the same field of view for a long time period (baseline is two long-pointing periods of two years each). ${ }^{1}$ However, PLATO has fixed solar panels orthogonal to the PLATO pointing direction, meaning that regular rotation of the satellite will be required in order to keep them at an acceptable view angle with the sun during the revolution of PLATO around the Sun, as highlighted in Fig. 2.

In order to maintain the exact same field of view before and after, these $90^{\circ}$ quarterly rolls impose some constraints both on the spacecraft operation and the payload design. Obviously, this concept works only if the $90^{\circ}$ rotation is performed around the pointing direction of PLATO payload, to ensure that the field of view before and after the turn remains the same. To achieve this, it is also required to have a full $90^{\circ}$ rotation symmetrical 


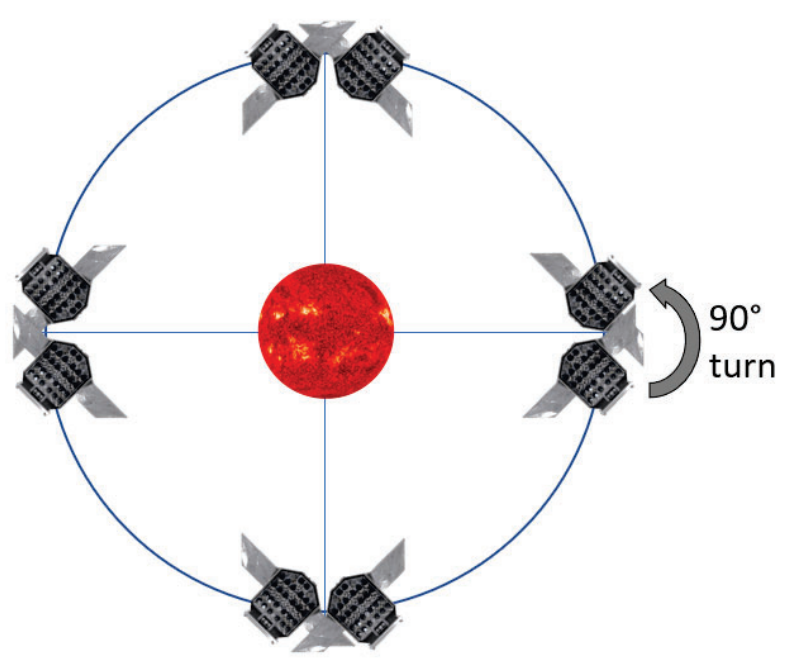

Figure 2. Operation concept of $90^{\circ}$ rotations around the pointing direction, 4 times per solar revolution (approximately every 3 months). Credits for the PLATO artist view: OHB System AG.

design of the whole payload. First, the camera arrangement on the Spacecraft has to be $90^{\circ}$ rotation invariant. This is the case as shown in Section 1.1, where each individual group field of view will overlap with its neighboring group field of view after a turn, see right figure in Fig. 1.

At camera level, the problematic is the same and the full design has to be rotational symmetric, in particular the Focal Plane Assembly (FPA) holding the CCDs. If this is not achieved, the consequence will be the loss of stars after a turn, that where observed in the previous 3 months observation period. Even if we obviously have 3 months of scientific data for these stars, we consider them as "lost" in the field of view budgets and in the stellar counts, because the scientific data doesn't meet all the scientific requirement of uninterrupted observation.

\subsection{Camera Design}

Each camera is split into 3 major sub-systems: a Telescope Optical Unit (TOU), a Focal Plane Assembly (FPA) and a Front End Electronic unit (FEE).

The TOU includes a total of 6 lenses that allow to create an image of a very large field of view of more than $18^{\circ}$ radius, see Refs. 3,4 for more details. A baffle is attached to the entrance protective window of the TOU to limit the straylight and also act as the main thermal radiator of the camera. The TOU is attached with 3 bipods to the FPA that offers a stable structure to support the 4 CCDs, described in Refs. 5, 6, that are needed to map the very large PLATO field of view. See Refs. 7 and 8 for more details on the FPA design. Finally, the FEE is mechanically attached to the TOU (for the N-CAM only) and linked to the FPA via the CCD flexis cables. This unit allows reading of the CCD and acquiring of images at the given cadence.

Section 3 presents in more details the focusing concept and budget of the camera, while Section 2 shows in details the field of view achieved by individual PLATO cameras and for the whole payload combined.

\section{FIELD OF VIEW BUDGET}

\subsection{Optical FoV}

As described in Refs. 3, 4, the Telescope Optical Unit (TOU) provide a circular FoV of around $18.8876^{\circ}$ radius, corresponding to the red circle in Fig. 3. This value includes the geometric distortion of less than $4 \%$ at the edge of this FoV. Actually, the optical FoV is slightly larger than that, but this value corresponds to the limit where the optical quality requirements are met. 
Even if this doesn't directly impact the FoV budget but more the SNR budget, it is worth mentioning that some vignetting is occurring inside this optical FoV. On top of the natural vignetting, there is some mechanical vignetting occurring at high FoV angles, caused by the fact that some lenses apertures were on purpose undersized to limit the mass of the cameras. This is controlled by a vignetting mask on the entrance window of the TOU. The total relative loss of photons at the edge of the FoV compared to on-axis is estimated to be around $17 \%$.

\subsection{Focal Plane Assembly}

The Focal Plane Assembly (FPA) has the main function of supporting the four CCDs needed per camera and maintain them in position with respect to the TOU. Its design is described in Refs. 7,8.

In terms of field of view budget, the FPA plays a major for PLATO's camera as the optical FoV is too large to be fully acquired on a single CCD. This means that several detectors (in this case 4 CCDs) have to be butted together, and will therefore create gaps within the optical FoV where no pixels will detect light.

As PLATO's field of view is one of the key parameters for science performance, it was decided to implement large enough CCDs to cover the whole "circle of light" provided by the TOU. This is a unique trade-off decision compared to the vast majority of optical cameras that generally implement smaller detectors to ensure that all pixels will be illuminated. A straylight mask is set on top of the four CCDs, fitting the optical FoV circle. This also means that a certain number of pixels per camera in each CCD corner will be covered under the straylight mask and will not be used for science measurements.
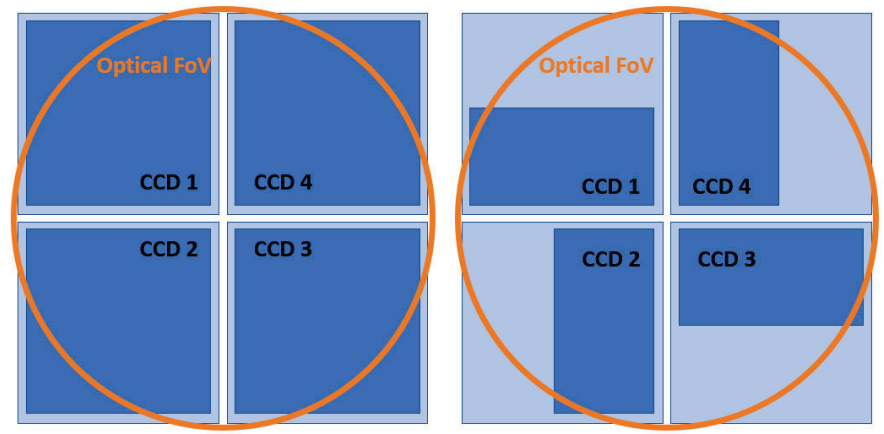

Figure 3. Optical FoV on top of the FPA geometry for both the N-CAM with full-frame CCD (left) and the F-CAM with frame-transfer CCDs (right). The dark blue section on the CCDs represent the actual die surface sensitive to light, the rest being the package or cover for the frame-transfer side.

Here are the relevant requirements that have to be taken into account for the field of view computation:

- The gap distance between the sensitive area and the package of each CCD shall be less than $1 \mathrm{~mm}$ (typical expected values are $1 \mathrm{~mm}$ in one direction and $0.4 \mathrm{~mm}$ the orthogonal one)

- The gap distance between $2 \mathrm{CCD}$ packages shall be less than $0.4 \mathrm{~mm}$

\subsubsection{Normal FPA Geometry}

The normal FPA geometry is presented on the left side of Fig. 3 with the red circle on top representing the optical FoV provided by the TOU.

To fully assess the impact of the FPA on the camera field of view budget, two main positions have to be taken into account: the position of each CCD package with respect to each other, and the position of the sensitive area of each CCD (dark blue area in Fig. 3) inside its own package (light blue area). Note that in order to distinguish misalignment from field of view loss, we define a "master CCD" (per definition CCD1). We then consider that any misalignment or position tolerances of this master CCD is counted in the internal camera pointing and 

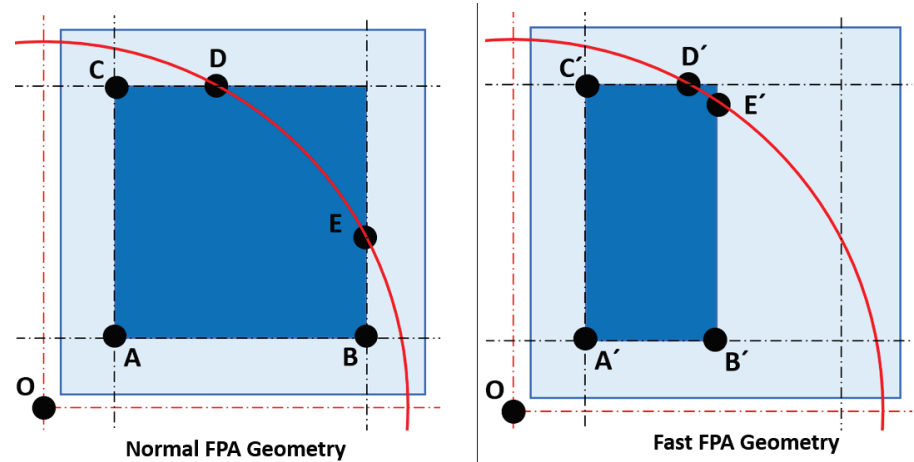

Figure 4. Detailed view of the N-FPA Geometry (left) and the F-FPA Geometry (right), focused on one of the four CCDs. The red arc represents the optical FoV, the light blue the package limit of the CCD and the dark blue the actual sensitive area. Sketch not to scale of the actual FPA.

alignment error budget (defined as having the camera pointing to the wrong field of view), while misalignment of the other CCDs compared to this master CCD enter the field of view budget described in this section.

Using the geometry defined in Fig. 4 and the different requirements on the CCD placement on the FPA, we can define the spatial coordinates for the main points A, B, C, D and E on the FPA:

Table 1. Coordinates of the points A, B, C, D, E defined in Fig. 4

\begin{tabular}{|l|l|l|l|l|}
\hline Point & $\mathrm{X}(\mathrm{mm})$ & $\mathrm{Y}(\mathrm{mm})$ & $\theta_{X}\left(^{\circ}\right)$ & $\theta_{Y}\left(^{\circ}\right)$ \\
\hline O & 0 & 0 & 0 & 0 \\
\hline A & 1.2 & 1.2 & $0.279^{\circ}$ & $0.279^{\circ}$ \\
\hline B & 82.38 & 1.2 & $17.868^{\circ}$ & $0.27^{\circ}$ \\
\hline C & 1.2 & 82.38 & $0.27^{\circ}$ & $17.868^{\circ}$ \\
\hline D & 30.41 & 82.38 & $6.757^{\circ}$ & $17.795^{\circ}$ \\
\hline E & 82.38 & 30.41 & $17.795^{\circ}$ & $6.757^{\circ}$ \\
\hline
\end{tabular}

The geometric coordinates in mm on the FPA are purely deduced from the requirements: $0.4 \mathrm{~mm}$ gap between $2 \mathrm{CCD}$ packages $+1 \mathrm{~mm}$ distance between the package and the sensitive area lead to a $1.2 \mathrm{~mm}$ translation in $\mathrm{X}$ and $\mathrm{Y}$ of the sensitive area compared to the optical axis $\mathrm{O}$ (assuming a perfect alignment). The sensitive area has 4510 pixels of $18 \mu \mathrm{m}$ each, meaning a total length of $81.18 \mathrm{~mm}$, that adds up to the $1.2 \mathrm{~mm}$ for a total of $82.38 \mathrm{~mm}$ with respect to the optical axis. These geometric values were used in OpticStudio Zemax with the camera optical model to determine the corresponding field of view values including the full distortion of the TOU.

The total field of view area corresponding to the area ABEDC in Fig.4 can then be computed by projecting it into the celestial sphere, to a total of $259.7 \mathrm{deg}^{2}$. Of course, to have the full field of view of a camera this value has to be multiplied by 4: $1038.8 \mathrm{deg}^{2}$. This value goes up to $1065 \mathrm{deg}^{2}$ if we consider typical values instead of requirements: optical FoV radius of $19.2^{\circ}$ and gap of the CCD sensitive area of $0.4 \mathrm{~mm}$ instead of $1 \mathrm{~mm}$ in one direction (1 $\mathrm{mm}$ remains in the other).

\subsubsection{Fast FPA Geometry}

The fast FPA geometry is presented on the right side of Fig. 3 with the red circle on top representing the optical FoV provided by the TOU.

Same as for the N-FPA case, both the position of each CCD package with respect to each other, and the position of the sensitive area of each CCD (dark blue area in Fig. 3) inside its own package (light blue area) are 
of interest. Again, we use the geometry defined in the right-hand side of Fig. 4 and the different requirements on the CCD placement on the FPA, we can define the coordinates for the main points A', B', C', D', E' and D':

Table 2. Coordinates of the points A', B', C', D', E' defined in Fig. 4

\begin{tabular}{|l|l|l|l|l|}
\hline Point & $\mathrm{X}(\mathrm{mm})$ & $\mathrm{Y}(\mathrm{mm})$ & $\theta_{X}\left(^{\circ}\right)$ & $\theta_{Y}\left(^{\circ}\right)$ \\
\hline O & 0 & 0 & 0 & 0 \\
\hline A $^{\prime}$ & 1.29 & 1.38 & $0.299^{\circ}$ & $0.32^{\circ}$ \\
\hline B $^{\prime}$ & 41.7 & 1.38 & $9.5^{\circ}$ & $0.317^{\circ}$ \\
\hline $\mathrm{C}^{\prime}$ & 1.29 & 82.2 & $0.289^{\circ}$ & $17.834^{\circ}$ \\
\hline $\mathrm{D}^{\prime}$ & 30.893 & 82.2 & $6.863^{\circ}$ & $17.759^{\circ}$ \\
\hline $\mathrm{E}^{\prime}$ & 41.7 & 77.281 & $9.228^{\circ}$ & $16.757^{\circ}$ \\
\hline
\end{tabular}

The geometric coordinates in mm on the FPA are purely deduced from the requirements: as for the N-FPA we consider $0.4 \mathrm{~mm}$ gap between 2 CCD Packages $+1 \mathrm{~mm}$ distance between the package and the sensitive area that again lead to a $1.2 \mathrm{~mm}$ translation in $\mathrm{X}$ and $\mathrm{Y}$ of the sensitive area compared to the optical axis $\mathrm{O}$ (assuming a perfect alignment). The sensitive area has 4510 pixels of $18 \mu \mathrm{m}$ each, meaning a total length of $81.18 \mathrm{~mm}$, that adds up to the $1.2 \mathrm{~mm}$ for a total of $82.38 \mathrm{~mm}$ with respect to the optical axis. However, the F-FPA is equipped with Frame-Transfer CCDs to allow a much faster readout of the image. To achieve this, half of the sensitive area is blocked by a metalization, leading to an effective sensitive area of 4490 x 2245 pixels. Indeed, on top of the $4510 \times 2255$ half that is blocked, a frame of respectively 5 pixels on each side in one direction and 10 pixels on each side in the orthogonal direction is also blocking the light, leading to this $4490 \mathrm{x} 2245$ pixels.

These geometric values were used in OpticStudio Zemax with the camera optical model to determine the corresponding field of view values including the full distortion of the TOU.

The total field of view area corresponding to the area A'B'E'D'C' in Fig. 4 can then be again computed by projecting it into the celestial sphere to a total of $153 \mathrm{deg}^{2}$. Of course, to have the full field of view of a camera this value has to be multiplied by 4: $612 \mathrm{deg}^{2}$. Again using typical values instead of requirements make this value increase up to $615 \mathrm{deg}^{2}$.

\subsection{Full Single CAM Budget}

Considering the values computed in the previous section, we can consider a total field of view of around 1038.8 square degrees for each N-CAM, and around 612 square degrees for each F-CAM.

These values are valid considering a perfect parallelism of CCD packages and sensitive areas. In practise, we expect up to 4 arcmin between the sensitive areas of CCDs 2, 3, 4 with respect to the package of CCD1 used as reference (CCD1 internal alignment is included in the camera alignment budget and not the field of view one). The total field of view lost because of 4 armin rotation of the sensitive area is less than 1 square degree, such as the impact of CCD shift from ground room condition to in-orbit cold environment expected to be less than $20 \mathrm{\mu m}$. The gap requirements of $2.4 \mathrm{~mm}$ in total are therefore the driving ones for this FoV budget.

We consider a total of around 1.5 to 2 arcmin lost because of misalignment and changes from ground to in-orbit conditions. We highlight again here that this part of the field of view is considered lost as it breaks the rotational symmetry of the design. The stars falling in these areas will be observed during 3 months, but then lost after the $90^{\circ}$ rotation and replaces by new stars.

Finally, we conclude that the estimated minimum field of view for the N-CAMs will be $1037 \mathrm{deg}^{2}$ and 610 $\operatorname{deg}^{2}$ for the F-CAMs. 


\subsection{Total budget}

As shown in Section 1.1, the $24 \mathrm{~N}-\mathrm{CAMs}$ are arranged in 4 groups of 6 cameras. This has been designed to maximize the SNR of the observed stars by overlapping the field of views of the cameras. Left side of Fig. 5 shows the final overlapping field of view of PLATO.

As mentioned directly in the figure, the central part of the field of view will be observed by all 4 groups and therefore a total of 24 cameras. Similarly, there are areas of the field of view observed by 3 groups or 18 cameras, and 2 groups or 12 cameras. Finally the edges of this geometric rosette will be only observed by the corresponding group pointing to that edge, and therefore only 6 cameras. The total estimated field of view is estimated to be approximately $2132 \mathrm{deg}^{2}$, with $294 \mathrm{deg}^{2}$ observed by 24 cameras, $171 \mathrm{deg}^{2}$ observed by 18 cameras, $796 \mathrm{deg}^{2}$ observed by 12 cameras, and $871 \mathrm{deg}^{2}$ observed by 6 cameras.
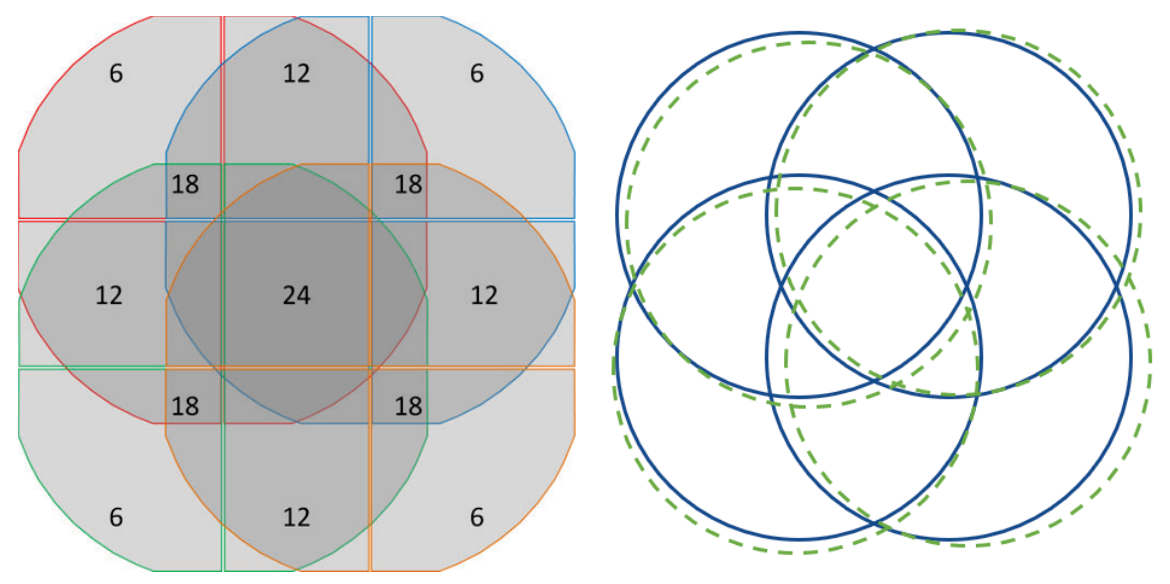

Figure 5. Left: Overlapping field of view of the PLATO payload. Each color represents a group field of view of 6 cameras, while the numbers show the number of camera observing each area of the FoV. Right: Example of the impact of misalignment of the groups on the overlapping FoV after a $90^{\circ}$ turn.

To ensure this overlap, the co-alignment of the cameras within one group has to be maximized. Considering the $90^{\circ}$ quarterly rotation, the co-alignment between groups is also very important for the field of view budget to ensure that group field of views before and after the rotation overlap perfectly, this is shown by the right side of Fig. 5. The blue circles represent the initial four group fields of view before the quarterly roll, and the dashed green ones the ones after the $90^{\circ}$ rotations. Any misalignment between the groups would result is a non-perfect overlap and a loss of field of view.

A budget of 6 arcmin half-cone angle absolute pointing error for each camera for the directional pointing and 13 arcmin for the rotational pointing is considered in the field of view computations presented above.

\section{FOCUSING BUDGET}

\subsection{Focusing Concept}

On top of this extraordinary large field of view presented in Section 2, PLATO's cameras have the uncommon feature of thermal refocusing capability. While each camera is designed to be mechanically athermal, the optical design has been performed such as to offer optical thermal refocusing.

The temperature of each camera is controlled by 3 heaters placed on its tube near the optical pupil. These heaters are commanded by a Proportional-Integral (PI) controller algorithm that regulates the camera temperature to very stable levels. By tuning these temperature heaters, the lenses inter-distances and refractive index will be modified leading to slight change of focus position. The expected thermal sensitivity of the optical focus is a linear change of at least $10 \mu \mathrm{m} / \mathrm{K}$. This will be used in-orbit to refocus each camera individually to its optimum focus temperature maximizing the SNR. 
Knowing that the cameras are designed to meet all their performance requirements at $-80^{\circ} \mathrm{C} \pm 10^{\circ} \mathrm{C}$, the total available focusing range available is of $\pm 100 \mu \mathrm{m}$. This focusing range has to be broken down into several contributors for each of the units and sub-systems involved in the camera, to then verify that the expected defocus is well within this range.

\subsection{TOU and FPA Focusing Budget}

The first part of the focusing budget is coming from the TOU, as it is the unit defining the initial optical best focus position. The main contributor for the focusing budget at TOU level is the characterization of the best image plane position at operational conditions $\left(-80^{\circ} \mathrm{C}\right)$ and the knowledge of its shift at room temperature where the integration will take place. More details on the best image plane characterization can be found in Royer at al (2020). ${ }^{9}$ The total allocation for the TOU is of $\pm 30 \mu \mathrm{m}$. It also includes smaller contributions such as tilt of the best image plane with respect to the interface reference frame and its stability from room to temperature to operational conditions.

Independently from the TOU, the FPA and especially the integration and alignment of the CCDs plays also a major role in the total focusing budget. Several contributors on the FPA can impact the focusing budget. The main one is the characterization of the FPA mean sensitive area position with respect to the interface reference frame. The accuracy of this characterization is estimated to be approximately $\pm 15 \mu \mathrm{m}$. Because of thermoelastic effect from cooling, gravity release and error in the model prediction of the warm-cold shift between the CCDs and the interface reference frame, we add a contribution of $\pm 7 \mu \mathrm{m}$. The total allocation for the FPA in the focusing budget is therefore of approximately $\pm 17 \mu \mathrm{m}$ by taking the Root-Square-Sum (RSS) of the 2 contributors above.

\subsection{CAM Integration}

As soon as the TOU and the FPA have been manufactured, aligned and tested, they will be delivered to the Centre Spatial de Liège (CSL) for integration and alignment, see Refs. 9,10. The main goal here is to bolt the FPA to the TOU at the right mechanical position, so that after cooling down of the CAM to operational temperature $\left(-80^{\circ} \mathrm{C}\right)$, the FPA Mean Sensitive Area lies on top of the optical best image plane. The total integration budget for the TOU-FPA alignment is set to $\pm 25 \mu \mathrm{m}$.

It has to be noted that the camera integration concept is still under refinement and that depending on the final chosen baseline, the allocations to the FPA and TOU might be affected. The budget presented here is meant as example based in the current baseline at the time.

\subsection{Final Budget}

\subsubsection{Calibration}

While all sub-contributions within each unit were combined with a Root-Square-Sum, we consider for the worstcase that they can add up linearly at system level, leading to a total of $\pm 72 \mu \mathrm{m}$. The meaning of this value is that, in the worst case, the the FPA Mean Sensitive Area can be up to $72 \mu \mathrm{m}$ away from the optical best focus position.

Considering a re-focusing range of $\pm 100 \mu \mathrm{m}$, this confirms that this budget allocation is compatible and should allow a fine refocusing of the cameras in operational conditions.

The integration and alignment of camera is performed fully at ambient laboratory conditions, and it is therefore impossible to verify that the actual camera defocus lies within the refocusing range and meets the performance specifications. For this purpose, an on-ground calibration of the best focus temperature is planned to be performed at operational conditions after the integration and alignment. As explained in Ref. 11, this calibration will allow to characterize the optical performance merit function of the camera as a function of its temperature. For each camera individually, the outcome of this test will be the nominal temperature of operation that ensure that we are operating the camera at its best focus, maximizing the scientific return. 


\subsubsection{Final In-Orbit performance}

The on-ground calibration will then be the starting point for a re-calibration in-orbit. Indeed, the on-ground environment can not perfectly reproduce the in-orbit environment, with for example gravity release effects, different thermal gradients inside the camera, or different thermal environment around it. Also, the Optical Ground Segment Equipment (OGSE) used on-ground is a collimator with a broadband source, that can not perfectly reproduce the stars observed by PLATO in-orbit. All these effects will add a couple of microns more to the focusing budget.

Finally, a contribution of $\pm 2 \mu \mathrm{m}$ contribution for the Thermal Control System (TCS) accuracy in-orbit is added, leading to a total of $\pm 76 \mu \mathrm{m}$ for the in-orbit focusing budget.

This value of $\pm 76 \mu \mathrm{m}$ is well inside the reachable focusing range of $\pm 100 \mu \mathrm{m}$ driven the $\left[-90^{\circ} \mathrm{C} ;-70^{\circ} \mathrm{C}\right]$ temperature range of operation, and offers enough margin in this early phase of the hardware development to be confident on the positive outcome.

On top of this, a total budget of $\pm 20 \mu \mathrm{m}$ is also in place to cover the non-correctable part of the defocus, mainly driven by the non-flatness of the CCDs on the FPA and their tilt with respect to the optical best image plane. These $\pm 20 \mu \mathrm{m}$ correspond to the acceptable depth of focus provided by the optical quality of the TOU.

\section{CONCLUSION AND OUTLOOK}

Thanks to its extraordinary large field of view and its in-orbit thermal refocusing capabilities, PLATO payload will be a truly unique instrument in space.

Indeed, with an estimated global field of view of $2132 \mathrm{deg}^{2}$ as shown in Section 2.4, PLATO will have by far the largest observing field of view in space. This is achieved using a combination of 26 cameras, spread in different groups with a $9.2^{\circ}$ angle between the group line of sights. To ensure that the overlap of the field of view of this cameras is optimal, very strict requirements are in place for every camera sub-units, but also on the camera alignment on the optical bench on the spacecraft.

On top of the quantity of targets reached thanks to this wide field of view, PLATO science requirements also specify very stable and high SNR observation of the stars. To overcome the classical problem of integration and alignment at ambient conditions and operation in cold vacuum environment, PLATO's cameras where designed such as to provide a thermal refocusing capability. With a total refocusing range of $\pm 100 \mu \mathrm{m}$, corresponding to a tunable operational temperature range of $\left[-90^{\circ} \mathrm{C} ;-70^{\circ} \mathrm{C}\right]$, the whole error due to the alignment and integration process of every camera sub-system and of the camera itself at laboratory ambient conditions can be covered and corrected in-orbit to maximize the science return of the PLATO payload.

\section{APPENDIX A. TRANSFORMATION OF COORDINATE MATRICES}

The rotation of the four N-Cam (nc) groups w.r.t. the F-Cams (fc) can be constructed using the quaternion representation as follows.

$$
\boldsymbol{q}_{n c}^{f c}=\left(\cos \left(\frac{\Theta}{2}\right), \boldsymbol{v} \sin \left(\frac{\Theta}{2}\right)\right)
$$

where $\Theta$ is the rotation angle, in this case 9.2 degree, and the rotation axis $\boldsymbol{v}=(x, y, 0)^{T}$, which is chosen for the four groups with

$$
\begin{aligned}
& \boldsymbol{v}_{1}=\left(-\frac{1}{\sqrt{2}}, \frac{1}{\sqrt{2}}, 0\right)^{T} \\
& \boldsymbol{v}_{2}=\left(-\frac{1}{\sqrt{2}},-\frac{1}{\sqrt{2}}, 0\right)^{T} \\
& \boldsymbol{v}_{3}=\left(\frac{1}{\sqrt{2}},-\frac{1}{\sqrt{2}}, 0\right)^{T} \\
& \boldsymbol{v}_{4}=\left(\frac{1}{\sqrt{2}}, \frac{1}{\sqrt{2}}, 0\right)^{T} .
\end{aligned}
$$


Since these axes are 90 degree rotation symmetric, the groups itself are 90 degree rotation symmetric. Applying the standard conversion to a rotation matrix leads to the following.

$$
\boldsymbol{R}_{n c}^{f c}=\left(\begin{array}{ccc}
\cos ^{2}\left(\frac{\Theta}{2}\right) & \operatorname{sgn}(x y) \sin ^{2}\left(\frac{\Theta}{2}\right) & \operatorname{sgn}(y) \frac{\sin (\Theta)}{\sqrt{2}} \\
\operatorname{sgn}(x y) \sin ^{2}\left(\frac{\Theta}{2}\right) & \cos ^{2}\left(\frac{\Theta}{2}\right) & -\operatorname{sgn}(x) \frac{\sin (\Theta)}{\sqrt{2}} \\
-\operatorname{sgn}(y) \frac{\sin (\Theta)}{\sqrt{2}} & \operatorname{sgn}(x) \frac{\sin (\Theta)}{\sqrt{2}} & \cos (\Theta)
\end{array}\right)
$$

\section{ACKNOWLEDGMENTS}

This work has benefited from financial support by DLR in the framework of its contribution to the PLATO Mission. This work presents results from the European Space Agency (ESA) space mission PLATO. PLATO data are being processed by the PLATO Mission Consortium (PMC). Funding for the PMC is provided by national institutions, in particular the institutions participating in the PLATO MultiLateral Agreement (MLA). The PLATO mission website is https://www.cosmos.esa.int/plato and Mission Consortium website is https://platomission.com/.

\section{REFERENCES}

[1] Rauer, H., Catala, C., Aerts, C., Appourchaux, T., Benz, W., Brandeker, A., Christensen-Dalsgaard, J., Deleuil, M., Gizon, L., Goupil, M.-J., and et al., "The plato 2.0 mission," Experimental Astronomy 38, 249-330 (Sep 2014).

[2] Ragazzoni, R., Magrin, D., Rauer, H., Pagano, I., Nascimbeni, V., Piotto, G., Piazza, D., Levacher, P., Schweitzer, M., Basso, S., Bandy, T., Benz, W., Bergomi, M., Biondi, F., Boerner, A., Borsa, F., Brandeker, A., Brändli, M., Bruno, G., Cabrera, J., Chinellato, S., Roche, T. D., Dima, M., Erikson, A., Farinato, J., Munari, M., Ghigo, M., Greggio, D., Gullieuszik, M., Klebor, M., Marafatto, L., Mogulsky, V., Peter, G., Rieder, M., Sicilia, D., Spiga, D., Viotto, V., Wieser, M., Heras, A. M., Gondoin, P., Bodin, P., and Catala, C., "PLATO: a multiple telescope spacecraft for exo-planets hunting," in [Space Telescopes and Instrumentation 2016: Optical, Infrared, and Millimeter Wave], MacEwen, H. A., Fazio, G. G., Lystrup, M., Batalha, N., Siegler, N., and Tong, E. C., eds., 9904, 731 - 737, International Society for Optics and Photonics, SPIE (2016).

[3] Magrin, D., Munari, M., Pagano, I., Piazza, D., Ragazzoni, R., Arcidiacono, C., Basso, S., Dima, M., Farinato, J., Gambicorti, L., Gentile, G., Ghigo, M., Pace, E., Piotto, G., Scuderi, S., Viotto, V., Zima, W., and Catala, C., "PLATO: detailed design of the telescope optical units," in [Space Telescopes and Instrumentation 2010: Optical, Infrared, and Millimeter Wave], Jr., J. M. O., Clampin, M. C., and MacEwen, H. A., eds., 7731, 715 - 722, International Society for Optics and Photonics, SPIE (2010).

[4] Magrin, D., "Plato telescope optical units: An update on working status," in [Space Telescopes and Instrumentation 2020: Optical, Infrared, and Millimeter Wave], Society of Photo-Optical Instrumentation Engineers (SPIE) Conference Series 11443-80 (2020).

[5] Endicott, J., Walker, A., Bowring, S., Turner, P., Allen, D., Piersanti, O., Short, A., and Walton, D., "Charge-coupled devices for the ESA PLATO M-class Mission," in [High Energy, Optical, and Infrared Detectors for Astronomy V], Holland, A. D. and Beletic, J. W., eds., 8453, 466 - 472, International Society for Optics and Photonics, SPIE (2012).

[6] Verhoeve, P., Prod'homme, T., Oosterbroek, T., Duvet, L., Beaufort, T., Blommaert, S., Butler, B., Heijnen, J., Lemmel, F., van der Luijt, C., Smit, H., and Visser, I., "Optical and dark characterization of the PLATO CCD at ESA," in [High Energy, Optical, and Infrared Detectors for Astronomy VII], Holland, A. D. and Beletic, J., eds., 9915, 356 - 371, International Society for Optics and Photonics, SPIE (2016).

[7] Moreno, J., Vielba, E., Manjón, A., Motos, A., Vázquez, E., Rodríguez, E., Saez, D., Sengl, M., Fernández, J., Campos, G., Muñoz, D., Mas, M., Balado, A., Ramos, G., Cerruti, C., Pajas, M., Catalán, I., Alcacera, M. A., Valverde, A., Pflueger, P., and Vera, I., "PLATO FPA. focal plane assembly of PLATO instrument," in [International Conference on Space Optics; ICSO 2018], Society of Photo-Optical Instrumentation Engineers (SPIE) Conference Series 11180, 111803N (July 2019). 
[8] Ramos, G., Guijarro, A. L. V., Rodrigo, M. T., Sierra, M. A., Gómez, L. J., Borreguero, E., Álvarez, L., Manjón, A., Balado, A., Barrado, D., and Mas, J. M., "Planetary transits and oscillation of stars (PLATO) focal plane assembly (FPA): prototype assembly and integration verification," in [UV/Optical/IR Space Telescopes and Instruments: Innovative Technologies and Concepts IX], Barto, A. A., Breckinridge, J. B., and Stahl, H. P., eds., 11115, 49 - 59, International Society for Optics and Photonics, SPIE (2019).

[9] Royer, P., L. Clermont, J., M. Pertenais, A. Baeke, Huygen, R., J. De Ridder, Regibo, S., M. Dami, Vandenbussche, B., A. Belen Balado Margeli, J. Farinato, D. Greggio, Y. Levillain, D. Magrin, L. Marafatto, M. Pajas, G. Ramos Zapata, A.L. Valverde Guijarro, and V. Viotto, "On the optical alignment of the PLATO cameras," in [Space Telescopes and Instrumentation 2020: Optical, Infrared, and Millimeter Wave], Society of Photo-Optical Instrumentation Engineers (SPIE) Conference Series 11443, 111443L (Aug. 2020).

[10] Clermont, L., Jacobs, J., Blain, P., Abreu, M., Vandenbussche, B., Royer, P., Huygen, R., Halain, J. P., and Baeke, A., "Automatized alignment of the focal plane assemblies on the PLATO cameras ," in [Space Telescopes and Instrumentation 2018: Optical, Infrared, and Millimeter Wave], Lystrup, M., MacEwen, H. A., Fazio, G. G., Batalha, N., Siegler, N., and Tong, E. C., eds., 10698, 1902 - 1913, International Society for Optics and Photonics, SPIE (2018).

[11] Pertenais, M., Cabrera, J., Griessbach, D., Erikson, A., Vandenbussche, B., Samadi, R., Reese, D., and Rauer, H., "Overview of PLATO's Cameras on-ground and in-orbit Calibration and Characterisation," Proc. SPIE XX, XX (2021). 